\title{
\%
}

Ana de Vicente Lancho*

Juan José Otamendi García-Jalón*

\section{ENTRADA EN VIGOR DEL ECO ¿CAMBIOS EN LA POLÍTICA MONETARIA DE ÁFRICA OCCIDENTAL?}

El eco se perfila como el sustituto del franco CFA para los ocho países que lo utilizan y como el embrión de una futura moneda común para los quince países que conforman la Comunidad Económica de África del Oeste. La estabilidad macroeconómica de esta moneda a lo largo de sus más de sesenta años de vida ha sido notable, especialmente comparada con los países de su entorno, aunque el crecimiento real de las economías que la utilizan no ha sido tan satisfactorio. Criticado por ser un vestigio colonial, se ha procedido a una vertiginosa reforma que suscita dudas sobre si se trata de un simple cambio de nombre o si va más allá y puede significar un giro en la política monetaria de la región en favor de un mayor crecimiento, pero que pueda poner en riesgo la estabilidad de la moneda y, con ello, su propia existencia.

Palabras Clave: unión monetaria, UEMOA, ECOWAS, CEDEAO, franco CFA.

Códigos JEL: E50, F45, F53, N17.

\section{Introducción}

El 21 de diciembre de 2019 el presidente francés, Emmanuel Macron, y el presidente de Costa de Marfil, Alassane Ouattara, realizaron una conferencia de prensa conjunta anunciando la sustitución del franco de la Comunidad Financiera Africana, moneda común de ocho países de África del Oeste, y avanzaron también que esa moneda sería el núcleo duro de la futura moneda de la CEDEAO (Comunidad Económica de África del Oeste). Esta iniciativa se explica, sobre todo, por la intención de

*Técnico Comercial y Economista del Estado.

Versión de septiembre de 2020.

DOI: https:/doi.org/10.32796/bice.2020.3129.7113
Francia de reducir su presencia en la gestión de la política monetaria en la región. No obstante, no está claro todavía si se trata de una serie de cambios puramente cosméticos o, por el contrario, será una revisión en profundidad que pueda llevar a un cambio en la política monetaria en la zona de la Unión Económica y Monetaria de África Occidental.

En este artículo revisaremos, en primer lugar, los antecedentes de esta moneda y las razones de la reforma, para pasar a evaluar la estabilidad macroeconómica de esta moneda respecto de otros países de la zona en los últimos veinte años, y terminar con la posible influencia que pueda tener tanto para África del Oeste como para su moneda hermana, el $\triangleright$ 
franco de la Comunidad Económica y Monetaria de África Central.

\section{Antecedentes: el franco de la Comunidad Financiera de África}

La creación del franco CFA original tuvo lugar en 1939, justo antes de la Segunda Guerra Mundial, aunque nació oficialmente en 1945, bajo el Gobierno provisional del general Charles de Gaulle. El objetivo inicial era reestablecer la autoridad monetaria francesa en estos territorios que habían quedado aislados durante el conflicto, catorce países de África subsahariana, que en ese momento eran aún colonias francesas. En ese momento, la abreviación CFA correspondía a «Colonias Francesas de África» y, entre 1958 y 1960, a «Comunidad Francesa de África». A partir de los procesos de independencia, se dividió entre el franco de la Comunidad Financiera de África en África del Oeste, y el franco de la Cooperación Financiera, en África Central, con sus respectivos institutos de emisión, que en 1959 dieron lugar al actual Banco Central de los Estados de África del Oeste (BCEAO) y al Banco Central de los Estados de África Ecuatorial y Camerún (que a partir de 1973 adoptó el nombre actual de Banco de los Estados de África Central, BEAC). Con el tiempo ${ }^{1}$ se crearon dos espacios económicos y monetarios: por un lado, la Unión Económica y Monetaria de África Occidental (UEMOA), que agrupa a Benín, Burkina

\footnotetext{
En puridad, la UEMOA se creó en 1994, pero la Unión Monetaria de África Occidental UMOA, creada en 1962, sigue existiendo y se ocupa de los aspectos monetarios de la zona, y el BCEAO se define como la institución monetaria de la UMOA, no de la UEMOA. Por su parte, el BEAC se define como el instituto de emisión de los Estados de la Comunidad Económica y Monetaria de África Central, creada en 1994 y en vigor desde 1999 y heredera de la Unión Aduanera Ecuatorial de 1959 y de su sustituta, la Unión Aduanera y Económica de África Central, creada en 1964, y en vigor desde 1966 hasta la creación de la CEMAC.
}

Faso, Costa de Marfil, Guinea-Bissau, Mali, Níger, Togo y Senegal; por otro, la Comunidad Económica y Monetaria de África Central (CEMAC) incluye a Camerún, República Centroafricana, Congo, Chad, Guinea Ecuatorial y Gabón. Estas dos monedas eran mutuamente convertibles (y también de cara al exterior) hasta que, en 1993, con las turbulencias previas a la devaluación de 1994, se suspendió esa convertibilidad entre las dos zonas, algo que se ha mantenido desde entonces.

Los principios rectores de ambas zonas monetarias han sido básicamente cuatro:

- Paridad fija con el franco y posteriormente con el euro.

- Libertad de flujos de capitales en la zona del franco CFA.

- Garantía de convertibilidad respaldada por el Tesoro francés.

- Centralización de reservas, a dos niveles. Por un lado, los Estados centralizaban sus reservas en los bancos centrales correspondientes, y por otro, depositaban el $50 \%$ de las mismas en una cuenta del Tesoro francés creada para cada uno de ellos.

El sistema funcionó razonablemente bien hasta finales de los años cincuenta, con saldos equilibrados y sin costes para Francia. Cuando comenzaron a independizarse las distintas colonias, surgió una primera ola de críticas de naturaleza ideológica, al ser el franco CFA un vestigio que mantenía la dependencia con la metrópoli. También algunos mandatarios consideraban una desventaja no tener un instrumento real de política monetaria, lo que explicó la salida del sistema de Malí en 1962 (aunque volvió a entrar en 1984 a 2 francos malienses por CFA cuando al salir la paridad era de $1 \triangleright$ 
a 1) y de Mauritania en 1973, o que Guinea ni siquiera llegara a entrar y emitiera desde la independencia su propio franco.

Otro tema de debate era el impacto de las fluctuaciones del franco francés, pues como consecuencia de las devaluaciones de la moneda francesa y del franco CFA, las importaciones en otras divisas se encarecían para los países africanos sin que el impacto en sus exportaciones se notase, al ser básicamente productos agrícolas (cacao, café, madera, algodón, plátano, etc.) con escasa elasticidad en el precio.

En 1973 se acordó una revisión del sistema, con la adopción, como principal medida de la reducción, del $100 \%$ al $65 \%$ de las reservas de divisas depositadas en el Tesoro francés. También se decidió «repatriar» las sedes de los bancos centrales africanos que se situaban en París.

La fuerte caída de los precios de las materias primas, a partir de 1985, hizo que se redujeran los ingresos por exportaciones de los países africanos, al tiempo que aumentaba la deuda exterior. En enero de 1994, bajo la presión francesa y con el acuerdo del Fondo Monetario Internacional (FMI), los catorce países africanos aceptaron devaluar el franco CFA un $50 \%$, pasando a la equivalencia 1 franco francés igual a 100 francos CFA, frente a los 50 hasta ese momento. Aunque la medida fue acompañada de apoyo a las exportaciones de materias primas, el impacto por la pérdida de poder adquisitivo en las economías africanas fue considerable. Hay que señalar que esta fue la única devaluación del franco CFA (para ambas zonas) desde su inicio hasta hoy en día. Además, a lo largo de su historia, se produjo una pequeña revaluación del franco CFA frente al de la metrópoli en 1948 y dos reajustes técnicos, con ocasión de la redenominación del franco francés, en 1960, y del lanzamiento del euro, en 1999. Por ello, diversos autores señalan respecto del papel de garante de Francia que cuando se enfrentó a la necesidad de hacer frente a su papel de garante, optó por la devaluación del franco CFA, aunque otros argumentan que sí que tuvo un coste para las arcas francesas (Strauss-Kahn, 2018).

\section{Evolución del franco CFA en el periodo 2000-2019}

Se ha escogido este intervalo por coincidir con la última etapa del franco CFA, cuando pasa a estar ligado al euro. Aunque esto pueda favorecer al franco CFA, ya que su única devaluación se produjo en 1994 y por lo tanto no se incluye, tampoco se tienen en cuenta las fuertes depreciaciones que se han producido en 2020 en el resto de monedas con el que se compara (de enero a agosto de 2020 , la naira nigeriana se ha depreciado un $28,2 \%$ y el cedi ghanés casi un 7\%).

El argumento más utilizado por los defensores del franco CFA ha sido, en realidad, el de la valoración positiva de un sistema de tipo de cambio fijo frente a uno flexible.

En el Gráfico 1 se recoge la pérdida de valor de las monedas de Ghana, Nigeria, Guinea, Gambia, Sierra Leona y Liberia respecto al euro. Si en el año 2000 se hubiera cambiado el equivalente a 100 euros en las respectivas monedas de esos países, el gráfico indica la cantidad de euros que se habrían obtenido si se hubiera optado por volver a transformar esas monedas en euros en cada uno de los años seleccionados. Así, por ejemplo, en el año 2000 , con 100 euros se hubieran obtenido 9.886 nairas. Al volver a cambiar esa cantidad de moneda doméstica a euros, habríamos $\triangleright$ 


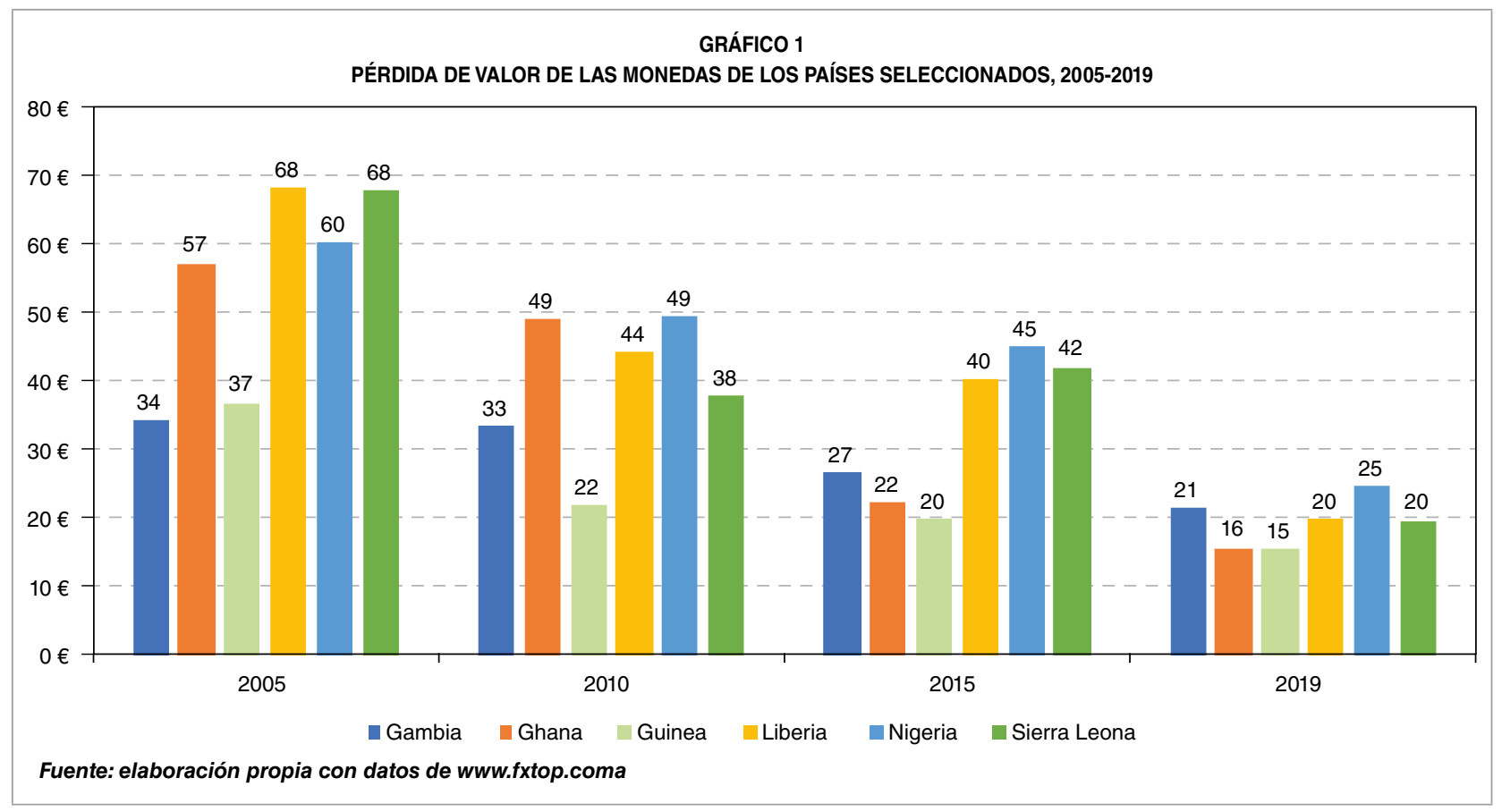

obtenido 60 euros si el cambio lo hubiéramos hecho en 2005 y 25 euros si lo hubiéramos hecho en 2019. Es decir, en este periodo la moneda nigeriana ha perdido el $75 \%$ de su valor frente al euro.

Esta es la tónica general para todas las monedas, que de hecho han perdido incluso más valor que la naira. En todos estos intervalos de cinco años, la pérdida de valor ha sido constante; únicamente el leone o chelín de Sierra Leona ha sido capaz de recuperar terreno frente al euro en una ocasión, ya que entre 2010 y 2015 se apreció ligeramente. Esta pérdida de valor además es asimétrica y perjudica más a los más pobres, que no tienen medios para cubrirse de ella.

Se puede argumentar que los países anglófonos tienen como moneda de referencia al dólar pero, en cualquier caso, las variaciones euro/dólar han sido muy pequeñas comparadas con las depreciaciones de estas monedas.

Como era de esperar, estas fuertes depreciaciones han traído consigo fuertes tasas de inflación, muy superiores a las de la zona UEMOA si atendemos al Cuadro 1, en el que se recogen las tasas de inflación y de crecimiento anuales medias entre 2000 y 2019 para los países seleccionados y también entre 2012 y 2019 (la razón para esto último es que entre 2002 y 2012 Costa de Marfil, que representa un tercio del producto interior bruto de la UEMOA, estuvo inmersa en sucesivas crisis político-militares que lastraron su economía).

De hecho, las tasas de la inflación de la UEMOA han sido consistentemente inferiores a las del resto de países de la región, ya que estos se sitúan, excepto Sierra Leona y Gambia (país rodeado en su totalidad por Senegal), en el entorno de los dos dígitos. Estas diferencias se acentúan más aún a partir de 2012.

En términos de crecimiento, los resultados son menos contundentes si consideramos todo el periodo 2000-2019. En efecto, el crecimiento de Sierra Leona lideraría el ranking con un $7 \%$, seguido del de Nigeria y Ghana, ambos rondando el $6 \%$, mientras que el de Guinea, $\triangleright$ 
CUADRO 1

TASAS DE CRECIMIENTO MEDIAS DEL PIBY LA INFLACIÓN EN LOS PERIODOS SELECCIONADOS

\begin{tabular}{|c|c|c|c|c|}
\hline & $\begin{array}{c}\% \text { crecimiento PIB real } \\
\text { 2000-2019 }\end{array}$ & $\begin{array}{c}\% \text { crecimiento PIB real } \\
\text { 2012-2019 }\end{array}$ & $\begin{array}{c}\text { Inflación media } \\
\text { 2000-2019 }\end{array}$ & $\begin{array}{c}\text { Inflación media } \\
\text { 2012-2019 }\end{array}$ \\
\hline UEMOA & 4,25 & 6,38 & 2,0 & 0,9 \\
\hline Gambia & 3,19 & 3,75 & 6,4 & 6,4 \\
\hline Ghana & 5,99 & 5,59 & 14,6 & 12,2 \\
\hline Guinea & 4,48 & 6,29 & 13,6 & 10,1 \\
\hline Liberia & 2,76 & 2,11 & 10,9 & 12,9 \\
\hline Nigeria & 6,07 & 2,74 & 12,0 & 11,6 \\
\hline Sierra Leona & 7,00 & 4,81 & 8,7 & 10,4 \\
\hline
\end{tabular}

$4,5 \%$, también sería dos décimas superior al del conjunto de la UEMOA, un $4,2 \%$, con lo que tendríamos que únicamente Gambia y Liberia han tenido un crecimiento más débil que el del bloque franco.

Ahora bien, estas conclusiones cambian si tenemos en cuenta únicamente los últimos siete años. En este subperiodo, el crecimiento de la UEMOA, un $6,4 \%$, es el mayor de todos, seguido muy cerca del de Guinea $(6,3 \%)$ y, a más distancia, del de Ghana (5,6\%). Destaca que el sólido crecimiento de Nigeria en la primera parte cae en los últimos ocho años por debajo de la tasa de crecimiento vegetativo, lo que lleva a un descenso en términos per cápita, que hace que su renta per cápita en 2019 (2.222 dólares) sea inferior a la de 2010 (2.328 dólares).

Estos resultados están en línea con los de Védie (2018), que considera que la solidez del franco CFA y su paridad fija es una importante ventaja que no ha perjudicado al crecimiento, y que cuando coyunturalmente este se ha visto negativamente afectado, se ha debido a otras circunstancias como disturbios o inseguridad. Además, menciona el argumento redistributivo de que la inflación penaliza sobre todo a los más pobres.

Otros argumentos son menos concluyentes, como el de que se favorece la inversión, al garantizar el tipo de cambio fijo del FCFA los retornos, ya que otros aspectos como la estabilidad política o la existencia de reservas petrolíferas parecen tener una mayor relevancia.

Sí parece claro que las relaciones comerciales en la región se han podido ver favorecidas por tener una moneda común, aunque de nuevo, en este caso, factores como la proximidad pueden ser igualmente relevantes. Como muestra el estudio de Goretti y Weisfeld (2006), la UEMOA tiene un comercio intrarregional mayor que el de cualquier otra zona de África, aunque es una cantidad pequeña, alrededor del $11 \%$.

Strauss-Kahn (2018), siguiendo a Severino, menciona como una de las características de la zona franco el bajo nivel de conflictos, aunque en nuestra opinión esto es más aplicable al siglo $x x$ que al actual, en el que ha habido muchos incidentes que han roto esta calma, desde la guerra en Costa de Marfil hasta golpes de Estado en Burkina, toma del poder por los islamistas en Mali o los problemas de terrorismo en el Sahel.

\section{El nacimiento del eco}

A pesar de este positivo comportamiento macroeconómico, las tasas de crecimiento $D$ 
de los países de la UEMOA han permitido que todos, excepto Costa de Marfil, sigan en la categoría de países menos adelantados (PMA). Esto ha generado una serie de debates, liderados por economistas africanos como Kako Nubukpo y Mamadou Coulibaly, que han ganado en intensidad desde 2015. Los críticos del franco CFA han insistido en que la paridad fija con el euro impide usar la política monetaria como instrumento que impulse la competitividad de las exportaciones (devaluación competitiva, que no tiene mucho sentido) y, en particular, consideran que la moneda está actualmente sobrevaluada en relación con la debilidad de las economías africanas. El resto de los argumentos se ha centrado en la influencia francesa en los órganos de decisión, la ventaja de Francia de mantener las reservas de los países miembros o incluso la dependencia que supone la impresión de los billetes en la antigua metrópoli.

En cambio, en el lado opuesto, los defensores del franco CFA argumentan que la impresión de una moneda en realidad requiere ciertas capacidades técnicas que no se tienen en África. De hecho, otras monedas fuera de esta zona son impresas en otros países europeos, como, por ejemplo, el franco guineano, el shilling de Uganda o el birr de Etiopía, que se imprimen en Reino Unido. Respecto a la utilización por el Tesoro francés de las reservas, el BCEAO tiene 32 cuentas en otras divisas como el yen, el dólar o la libra en otros países y, en todo caso, son cuentas remuneradas por Francia, al $2 \%$ en la actualidad. Dominique StraussKhan en 2018 ya abogaba por una emancipación del franco, una evolución del sistema, pero advertía en contra de dos tentaciones: la primera, limitarse únicamente a los símbolos, es decir, hacer una reforma puramente cosmética; la segunda, llevar a cabo una reforma radical.
En todo caso, haciendo frente a algunas de estas críticas, Francia y los miembros de la UEMOA han decidido reformar determinadas condiciones de la zona monetaria.

El FMI emitió un comunicado saludando la reforma, al considerar que constituye un paso en la modernización de los acuerdos entre la zona UEMOA y Francia, pero que se mantienen las claves de la estabilidad macroeconómica que ha disfrutado la región.

El 20 de mayo de 2020 Francia empezó el proceso para aprobar oficialmente el final del franco CFA, al menos el de la zona oeste, a través de un proyecto de ley a este fin que todavía no se ha aprobado². Los tres cambios que se aprueban con esta norma son:

- El cambio de nombre de la moneda de la UEMOA, que pasa a ser el eco.

- El Banco Central de los Estados de África del Oeste (BCEAO) no tendrá que depositar la mitad de las reservas en el Tesoro francés.

- La retirada de Francia de las instancias de gobierno en las que estaba presente, concretamente del Consejo de Administración, del Comité de Política Monetaria de la BCEAO y de la Comisión bancaria de la UEMOA.

A partir de ahora el papel de Francia no será más que el de un garante financiero. En efecto, si la BCEAO tiene necesidad de divisas para hacer frente a sus compromisos, podrá obtener de Francia los euros necesarios ${ }^{3}$.

Sin embargo, es posible que todo cambie para que nada cambie. En el proyecto de ley $\triangleright$

\footnotetext{
2 Se encuentra en la fase de revisión en la Comisión de Asuntos Exteriores del parlamento francés (http://www.assemblee-nationale.frl dyn/15/textes/115b2986_projet-loi).

3 Esta situación, recordemos, no se ha producido más que una vez en sesenta años de existencia del FCFA y nunca en los últimos veinticinco años.
} 
se menciona, en su artículo 2, la garantía de convertibilidad de la moneda por parte de Francia, pero precisa que una convención de garantía entre el ministro de Finanzas francés y el gobernador de la BCEAO establezca las modalidades de activación. El artículo 10, por su parte, establece que la entrada en vigor del acuerdo de cooperación entre el Gobierno francés y los Gobiernos de la UEMOA se producirá cuando entre en vigor la convención de garantía que se menciona en el artículo 2. De igual forma, aunque la labor de los representantes franceses en el Consejo de Administración y en el Comité de Política Monetaria de la BCEAO, y en la Comisión bancaria de la UMOA, cesará ese mismo día, el artículo 4 prevé el nombramiento por el Consejo de Ministros de la UMOA de una persona cualificada para el Comité de Política Monetaria en concertación con la República Francesa; el artículo 5 establece el envío regular de información técnica del BCEAO a Francia para que pueda hacer un seguimiento de las garantías que cubre y del principio de reuniones técnicas si son necesarias; el artículo 8 dispone que la convención de garantía establecerá las modalidades de colaboración temprana en caso de crisis y fijará también el retorno de un representante de Francia al CPM en caso de crisis aguda, que se establece en una ratio entre los activos exteriores de la BCEAO y sus obligaciones a la vista igual o inferior al $20 \%$.

Quedan algunas cuestiones pendientes de definir, pues aún no ha quedado claro cuándo se hará la sustitución efectiva de los billetes del FCFA por los del eco. Tampoco se sabe en qué momento esta reforma será completada con la adopción del eco por toda la CEDEAO, o al menos por algún país más (Strauss-Kahn señala a Ghana como primer paso). En la 57. a $^{-}$ sesión ordinaria de la Conferencia de jefes de Estado y de Gobierno de la CEDEAO, del 5 de septiembre de 2020, se acordó, entre otras cuestiones: (i) elaborar una nueva hoja de ruta para el Programa de Moneda Única; (ii) diferir a una fecha ulterior el lanzamiento de la moneda única; (iii) eximir a los Estados miembros del respeto de los criterios de convergencia macroeconómica en 2020; (iv) concluir, entre los Estados miembros de la CEDEAO, un nuevo pacto de convergencia y de estabilidad macroeconómica; y (v) mantener el enfoque gradual para el lanzamiento del eco. En unas declaraciones realizadas por el presidente marfileño Alassane Ouattara tras esa reunión, apuntó que, al menos, serán necesarios de tres a cinco años para que el eco vea la luz como moneda fiduciaria. Además, hay que tener en cuenta que el nacimiento del eco estaría siempre sujeto a la satisfacción de ciertos criterios de convergencia: un déficit presupuestario inferior al $3 \%$ del PIB, una tasa de inflación por debajo del 10\% y un volumen de deuda pública que no rebase el $70 \%$ del PIB, lo que, según el informe de convergencia de la CEDEAO, en 2019 no ha sido respetado por ninguno de los países miembros.

\section{Consecuencias de la creación del eco}

Podemos dividirlas en los efectos para la política monetaria de la actual zona CFA de África del Oeste, los efectos que pueda tener sobre la moneda única de la Comunidad Económica de Estados de África Occidental (Ecowas) y, en tercer lugar, el efecto imitación que puede darse en la otra zona del franco CFA, la de África Central.

Respecto de la primera de ellas, aunque las autoridades francesas, el FMI y los países implicados han repetido que las líneas $\triangleright$ 
generales de la política monetaria no van a cambiar, existen dudas sobre la credibilidad de la paridad ante la gran probabilidad de una posible devaluación. En todo caso, la confianza tiene un valor especial en los mercados de divisas y financieros, y quebrarla con cambios poco estructurados puede tener más desventajas que ventajas en una zona donde la estabilidad de la llegada de capitales es clave para el desarrollo. A finales de febrero de 2020, la agencia S\&P realizó un estudio sobre el proyecto y concluía que mientras siguiera anclada al euro y con Francia como garante de su convertibilidad, no veía motivos de preocupación, y descartaba una devaluación.

Sin embargo, es más que dudoso que los críticos con el franco acepten este simple cambio cosmético, ya que su énfasis ha sido siempre el de llevar a cabo una política monetaria mucho más expansiva, argumentando que, de esta forma, se conseguirán unas tasas mayores de crecimiento que puedan sacar a estos países de la situación de estancamiento en la que se encuentran, puesto que todos los países de la región, con la excepción de Nigeria, Ghana y Costa de Marfil, tienen la categoría de países menos adelantados (PMA). En línea con los postulados de la teoría monetaria moderna y envalentonados por este primer cambio, seguirán insistiendo en la necesidad de una expansión monetaria en la zona franco, sin tener en cuenta que, en los países africanos con autonomía monetaria, esto ya se ha intentado y los resultados han sido, por lo general, no muy positivos, no ya solo en la región de África del Oeste, sino también en otros países como Zimbabue.

Por su parte, los Gobiernos, que hasta ahora siempre podían argumentar que tenían las manos atadas, tendrán más difícil resistir presiones en favor de políticas más expansivas, máxime en un entorno global en el que los países desarrollados están ya recurriendo, de forma generalizada, a medidas expansivas del tipo quantitative easing.

Estas dudas sobre si la zona mantendrá la ortodoxia monetaria ante la difícil coyuntura que se abre en la actualidad para la región, tras el impacto de la crisis sanitaria del coronavirus, se ven acrecentadas bajo el enfoque de una moneda común en la CEDEAO, que incluiría a algunos países con una reputación inflacionaria dudosa, como Ghana o Nigeria. Si Ghana entrara en el eco, existirían fuertes presiones para que la política monetaria común fuera mucho más expansiva, ya que Ghana puede argumentar que le ha funcionado relativamente bien esa receta, puesto que, como hemos visto, a pesar de la fuerte pérdida de valor de su moneda y de la mayor inflación, lo cierto es que sus tasas de crecimiento han sido superiores en el periodo 2000-2019 y, aun cuando han sido inferiores en los últimos ocho años, lo han sido únicamente de forma marginal. Mayor problema supondría la inclusión de Nigeria, cuyo peso en la economía de la CEDEAO roza el $70 \%$. Es mucho más lógico pensar que la política monetaria se adaptará al gigante de la zona que lo contrario: importar la credibilidad antiinflacionista de la zona CFA de forma rápida y sin coste, como hicieron los países periféricos con Alemania en el nacimiento del euro, es más que utópico, irreal.

Precisamente una segunda consecuencia del nacimiento de este eco, a la que hay que prestar atención, es la interferencia que pueda causar en el proyecto de moneda única de la Comunidad Económica de Estados de África del Oeste (CEDEAO/Ecowas) ${ }^{4}$. Este es un $\triangleright$

4 Fundada en 1975, agrupa además de los países de la UEMOA a otros siete países: Gambia, Ghana, Guinea, Liberia, Nigeria, Sierra Leona y Cabo Verde (los seis primeros forman parte de una zona monetaria de 
antiguo proyecto, inicialmente planeado para el año 2000 y que se ha ido posponiendo. En su cumbre de julio de 2019, en Abuya, se acordó llamar a esta futura moneda precisamente de igual forma, eco (incluso se aventuraron a prever la fecha del 1 de julio de 2020 para su lanzamiento). Por ello, Nigeria considera que esta iniciativa se ha llevado a cabo de una forma precipitada y que se debería inscribir en el proyecto más amplio de una moneda común para el conjunto de la CEDEAO. En este sentido, el presidente nigeriano ha insistido en que la situación está lejos de permitir cumplir los criterios de convergencia mínimos que asegurarían el éxito de la adopción de una moneda común. El 16 de enero de 2020 los países de la Zona Monetaria de África Occidental (ZMAO), es decir, Gambia, Ghana, Guinea, Liberia, Nigeria y Sierra Leona, criticaron la decisión de que el franco CFA pasara a llamarse eco, e insistieron que la moneda de Ecowas seguiría adelante ese mismo año siempre que se cumplieran cinco criterios, que Nigeria considera que no se daban, por lo que habría que aplazarla. Ghana, por su parte, se ha mostrado partidaria de unirse al eco siempre y cuando se abandone la paridad fija con el euro.

Por lo que respecta a los países de la CEMAC, este proceso de creación del eco parece que les ha animado a hacer sus propios cambios. En una cumbre extraordinaria, celebrada en noviembre de 2019, declararon su disposición a reformar su acuerdo monetario con Francia y su urgencia, a lo que esta ha respondido que está dispuesta. En una visita en diciembre a Costa de Marfil, el presidente de Guinea Ecuatorial calificó al franco CEMAC de obsoleto, y posteriormente en esa línea se

África del Oeste, ZMAO, creada en 2000). Tiene como objetivo promover la cooperación y la integración económica entre sus miembros y, desde 1993, también avanzar hacia la unidad financiera y monetaria. manifestaron varios dirigentes de la región. Aunque no se ha avanzado debido a la pandemia de coronavirus que afecta a todo el mundo desde mediados de 2020, ya se ha mencionado incluso un nombre, el afrix, y, por lo tanto, una reforma en la misma línea que sus homólogos del oeste se puede dar por hecha, y lo dicho anteriormente es, asimismo, de aplicación a este caso.

\section{Conclusión}

Aunque ha habido algunos cambios recientes, todavía hay mucha confusión y falta de información sobre el proyecto de reforma de la moneda única en la CEDEAO. Ni el alcance, en cuanto a los países implicados, ni las fechas aproximadas para configurar un calendario razonable parecen estar definidos. Además, el empeoramiento de la situación económica global y en África en particular, como consecuencia de la crisis sanitaria desencadenada por la COVID-19, hace alejar aún más la perspectiva de que el proyecto avance a corto plazo.

En cualquier caso, a pesar de la progresiva reducción de la influencia francesa en la política monetaria de la UEMOA, la mayoría de los analistas abogan por que se mantengan las líneas principales de actuación seguidas en los últimos veinticinco años, que han permitido una considerable estabilidad macroeconómica. Sin embargo, no hay que descartar que los críticos con el sistema anterior no se conformen con algunos pequeños cambios y quieran profundizar en una mayor autonomía monetaria para la zona franca que termine siendo perjudicial para esa tradicional estabilidad. Si a esto añadimos la posible incorporación a esta moneda de otros países con una peor reputación inflacionista, el riesgo aumenta considerablemente, no tanto $D$ 
Ana de Vicente Lancho y Juan José Otamendi García-Jalón

a corto plazo, dado que es difícil que otros países cumplan los criterios de convergencia establecidos para unirse a esa moneda común, pero sí a medio y largo plazo.

\section{Bibliografía}

Agbohou, N. (2016). Le franc CFA et l'euro contre l'Afrique. Éditions Solidarité Mondiale.

Avom, D., y Noumba, I. (2019). La résilience de la zone Franc à l'épreuve des critiques persistentes. Revue Internventions Economiques, 61/ 2019. https://doi.org/10.4000/interventionseconomiques.5466

BCEAO (2017). Chronologie des évènements marquants de l'histoire de la BECEAO et de I'UEMOA. https://www.bceao.int/fr/publications/ chronologie-des-evenements-marquants-deIhistoire-de-la-bceao-et-de-lumoa

Goretti, M., y Weisfeld, H. (2006). Trade in the WAEMU: Developments and Reform Opportunities. IMF Working paper WP/08/06.

Guillaumont Jeanney, S., y Guillaumont, P. (2013). Les accords de coopération monétaire de la zone Franc : atouts et contraintes. Document de travail de la Fondation pour les Études et Recherches sur le Développement International (65).

Guillaumont Jeanney, S., y Guillaumont, P. (mayo 2017). Quel avenir pour les francs CFA? FERDI working document.

Guillaumont Jeanney, S., y Guillaumont, P. (2017). La zone Franc en perspective. Revue d'Économie du Développement, 25 (2), 5-40. DOI : 10.3917/edd.312.0005

Jacqueront, P. (2017). Faut-il tuer le franc CFA? Institut de relations Internationales et Stratégiques. https://www.iris-france.org/102197-faut-il-en-finiravec-le-franc-cfa-2/

Nubukpo, K., y otros autores (2017). Sortir de l'Afrique de la servitude monétarie. A qui profite le franc CFA? La Dispute.

Strauss-Kahn, D. (2018). Franc Zone: for an emancipation benefitting all. https://es.slideshare.net/ DominiqueStraussKahn/franc-zone-for-an-eman cipation-benefitting-all

Vedié, H. (2018). Le franc CFA : impact sur les taux d'inflation et de croissance des pays d'Afrique de l'Ouest et d'Afrique centrale sur la période 1999-2017, OCP Policy Center. 